\title{
Studying the Bioactivity of Tissue Engineering Scaffolds Derived from Egg and Sea Shell Waste Using SEM, EDS, \& TEM
}

\author{
Vincent Hembrick-Holloman ${ }^{1 *}$, Vijaya K. Rangari ${ }^{1}$, Temesgen Samuel ${ }^{2}$ and Shaik Jeelani ${ }^{1}$ \\ 1. Department of Material Science and Engineering, Tuskegee University, Tuskegee, AL, USA. \\ 2. Department of Pathobiology, College of Veterinary Medicine, Nursing and Allied Health, Tuskegee \\ University, Tuskegee, AL, USA. \\ * Corresponding author: vholloman4506@tuskegee.edu
}

In the biomedical field, there is an urgent need for regenerative, biocompatible materials that can be fabricated into complex and patient-specific shapes. In this study, engineered nano hydroxyapatite (nHA) was derived from naturally occurring chicken eggshell and clam sea shell waste using an energy efficient microwave-assisted wet chemical precipitation method.

Eggshell waste is produced at a rate of 8 million tons per year worldwide and the disposal of this massive amount of waste is a very environmentally challenging issue [1]. Seashell waste is another waste material that is available in abundance. China alone produces 10 million tons of shell fish waste that is disposed of annually. This sea shell waste primarily consists of oyster, clam, scallop, and mussel shells which are mostly wasted in landfills with only a very small fraction being re-used for other purposes.

Instead of simply aiming to dispose of this waste, it can be converted into new materials that can be utilized for several different types of applications [2, 3]. Eggshell and seashell waste can be utilized because they serve as natural sources of calcium carbonate $\left(\mathrm{CaCO}_{3}\right)$ and can be converted into valuable bioactive materials. The high amount of calcium carbonate was used in this study as a source of calcium ions for the synthesis of the calcium phosphate-based material known as hydroxyapatite.

The objective of this research is to develop a unique and sustainable approach to fabricate complex 3D scaffolds for tissue engineering. Various methods have been used to fabricate 3D scaffolds for tissue regeneration. However, conventional techniques such as salt leaching, freeze-drying, gas forming, and phase separation don't allow precise control of the internal scaffold architecture or the fabrication of complex architectures. These limitations make 3D printing technology an attractive alternative.

Using 3D printing to fabricate objects layer by layer to create a $3 \mathrm{D}$ volumetric structure allows the ability to control scaffold architecture, internal pore structure, and fine tune the properties of biomaterial based scaffolds [4]. Limitations still exist with 3D printing which includes the limited number of biomaterials that can be processed and fabricated compared to the conventional techniques [5]. The nHA infused polymer scaffolds were fabricated using slurry-based solution 3D printing techniques. To our knowledge this is the first 3D printing technique that can fabricate complex hydroxyapatite-based scaffolds with a weight percentage of up to $70 \%$.

Analysis of nHA particles were performed using transmission electron microscopy (TEM) JEOL Model JEM-2010, scanning electron microscopy (SEM) JEOL Model JSM-7200F coupled with energy dispersive spectrometer (EDS) EX-230, for determination of particle shape, morphology and elemental composition. Scanning electron microscopy was also used to investigate the interactions between 3D 
printed HA scaffolds and fibroblast cells over various time frames. SEM, TEM, and EDS analyses show that the particles are highly crystalline HA with acicular shapes, and in nanometer size ranges. The scaffolds derived from naturally occurring waste sources showed high levels of cellular adhesion and proliferation when seeded over various time frames as compared to other sources.

\section{References:}

[1] G De Angelis et al., J. Clean. Prod. 164 (2017), p. 1497.

[2] KH Mo et al., Constr. Build. Mater. 162 (2018), p. 751.

[3] Z Yao et al., Crit. Rev. Environ. Sci. Technol. 44 (2014), p. 2502.

[4] QL Loh and C Choong, Tissue Eng. Part B Rev. 19 (2013), p. 485.
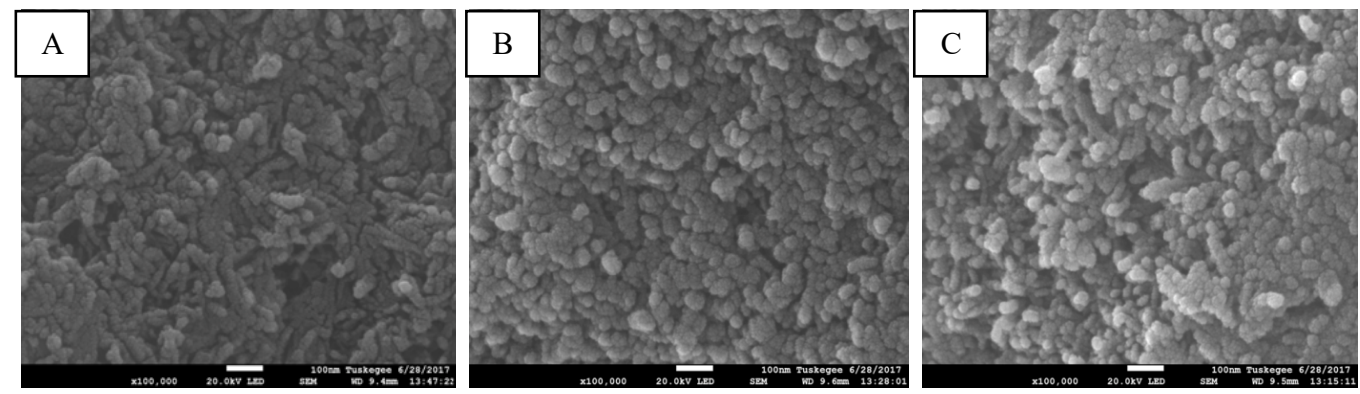

Figure 1. FESEM micrographs showing as-synthesized (a) ESnHA, (b) LNnHA, (c) QSnHA.
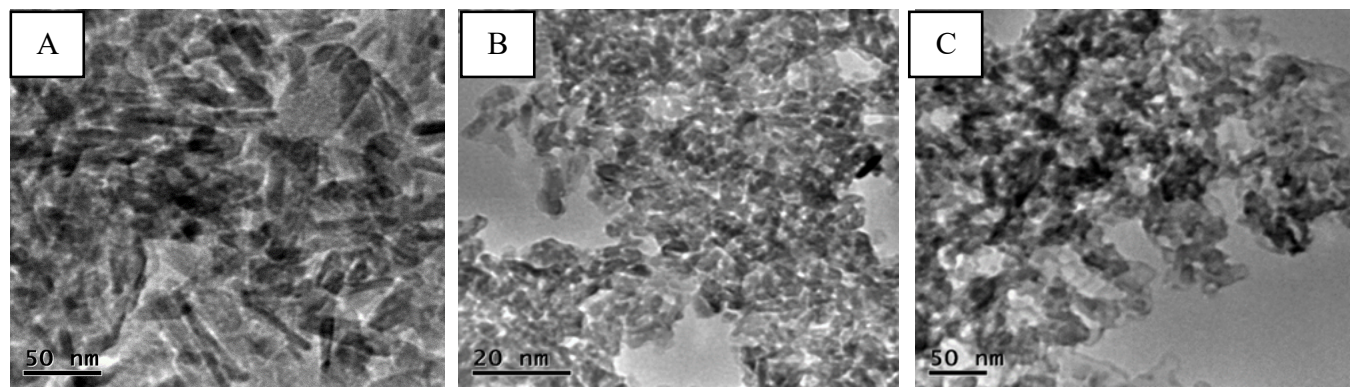

Figure 2. HRTEM micrographs showing as-synthesized (a) ESnHA, (b) LNnHA, (c) QSnHA.
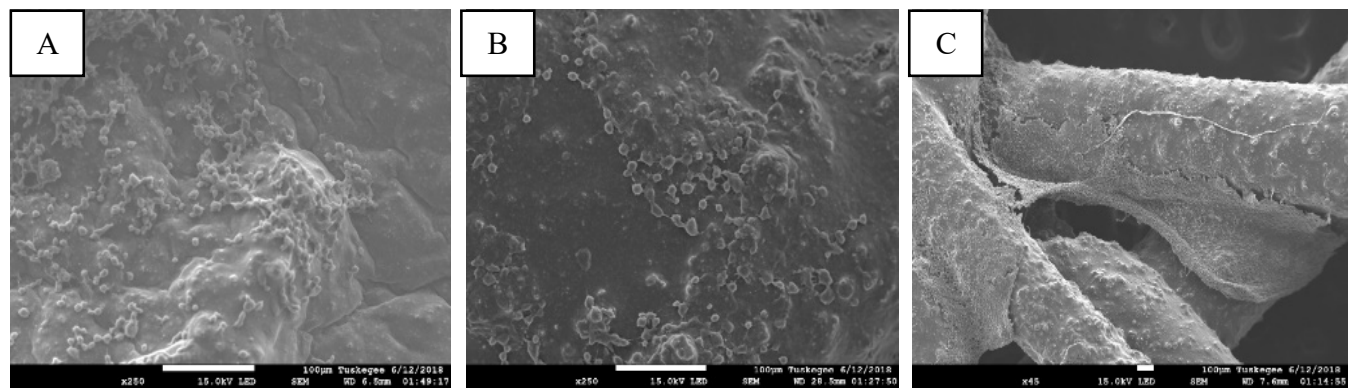

Figure 3. SEM micrographs showing cell interactions on nHA based scaffold surfaces after 1 day. 